\title{
The running fine structure constant $\alpha(E)$ via the Adler function
}

\author{
F. Jegerlehner ${ }^{\mathrm{ab} * \mathrm{c} \dagger}$
}

${ }^{a}$ H. Niewodniczanski Nuclear Physics Institute PAN, 31-342 Krakow, Poland

${ }^{\mathrm{b}}$ Humboldt-Universität zu Berlin, Institut für Physik, Newtonstrasse 15, D-12489 Berlin, Germany

${ }^{\mathrm{c}}$ Deutsches Elekronen-Synchrotron DESY, Platanenalle 6, D-15738 Zeuthen, Germany

We present an up-to-date analysis for a precise determination of the effective fine structure constant and discuss the prospects for future improvements. We advocate to use a determination monitored by the Adler function which allows us to exploit perturbative QCD in an optimal well controlled way. Together with a long term program of hadronic cross section measurements at energies up to a few $\mathrm{GeV}$, a determination of $\alpha\left(M_{Z}\right)$ at a precision comparable to the one of the $Z$ mass $M_{Z}$ should be feasible. Presently $\alpha(E)$ at $E>1 \mathrm{GeV}$ is the least precisely known of the fundamental parameters of the SM. Since, in spite of substantial progress due to new BaBar exclusive data, the region 1.4 to $2.4 \mathrm{GeV}$ remains the most problematic one a major step in the reduction of the uncertainties are expected from VEPP-2000 [1] and from a possible "high-energy" option DAFNE-2 at Frascati [2]. The up-to-date evaluation reads $\Delta \alpha_{\text {had }}^{(5)}\left(M_{Z}^{2}\right)=0.027515 \pm 0.000149$ or $\alpha^{-1}\left(M_{Z}^{2}\right)=128.957 \pm 0.020$.

\section{INTRODUCTION}

The accuracy of theoretical predictions of precision observables often is limited as soon as low energy hadronic physics comes into play. In fact, one of the main non-perturbative hadronic effect contributing to many electroweak precision observables is the hadronic vacuum polarization which affects the effective fine structure "constant" $\alpha(E)$. For precise SM predictions one thus needs to know the running $\alpha$ very precisely. As $\alpha(E)$ is steeply increasing at low $E$, substantial corrections show up at low scales already. Furthermore, in the time-like region, nonperturbative resonance effects make $\alpha(E)$ to be a complicated function, as illustrated in Fig. 1.

\section{2. $\alpha\left(M_{Z}\right)$ IN PRECISION PHYSICS}

For SM predictions the most precisely known parameters $\alpha, G_{\mu}$ and $M_{Z}$ are chosen as the basic input parameters. However, for processes be-

\footnotetext{
*Permanent address.

†Work supported in part by the EU grants MTKDCT2004-510126 in partnership with the CERN Physics Department and with the TARI Program under contract RII3-CT-2004-506078.
}

yond the very low energy region not $\alpha$ itself but $\alpha(E)$ plays the role of $\alpha$. This has dramatic consequences for precision physics: the uncertainties of the hadronic contributions to the effective $\alpha$ represent a major limitation for electroweak precision physics. In fact $\alpha(E)$ above about $1 \mathrm{GeV}$ is a factor of 10 less well known than the next worse which is the $Z$ mass $M_{Z}$. This is particularly important for a precise investigation of $Z$ and $W$ gauge boson physics, for example. The present accuracies of the main SM parameters read $\delta \alpha / \alpha \sim 3.7 \times 10^{-10}, \delta G_{\mu} / G_{\mu} \sim 8.6 \times 10^{-6}$, $\delta M_{Z} / M_{Z} \sim 2.4 \times 10^{-5}$, but $\delta \alpha\left(M_{Z}\right) / \alpha\left(M_{Z}\right) \sim$ $1.1 \div 2.6 \times 10^{-4}\left[\delta \alpha(E) / \alpha(E) \sim 4.5 \times 10^{-5}\right.$ at $E=1 \mathrm{GeV}$ space-like]. Thus at present we loose a factor $10^{5}$ in precision in the replacement $\alpha \rightarrow \alpha\left(M_{Z}\right)$. For precision physics at the ILC one would require $\alpha\left(M_{Z}\right)$ to be determined as precise as $M_{Z}$ [3], typically, which would require an improvement by a factor about 10 to obtain

$$
\frac{\delta \alpha\left(M_{Z}\right)}{\alpha\left(M_{Z}\right)} \sim 2.5 \times 10^{-5} .
$$

At present, an important example is the LEP/SLD measurement of $\sin ^{2} \Theta_{\text {eff }}=(1-$ $\left.g_{V l} / g_{A l}\right) / 4=0.23148 \pm 0.00017$ from which the Higgs mass bound depends most sensitively. An 

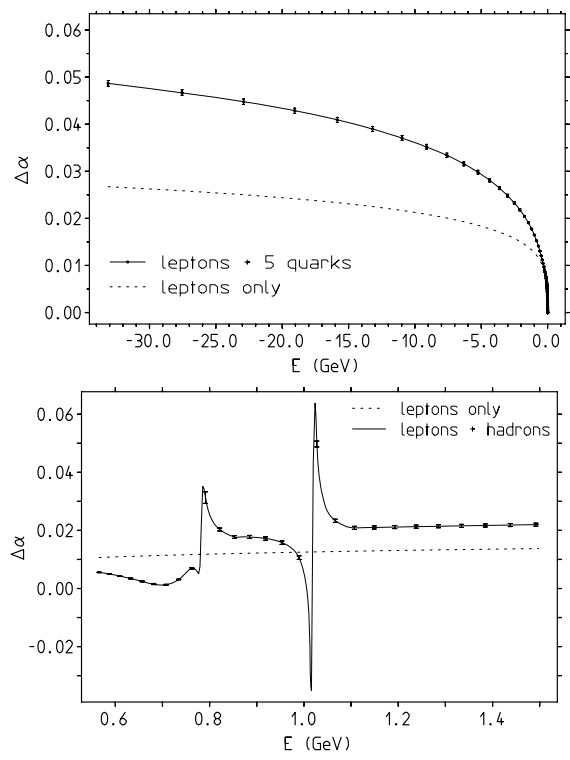

Figure 1. The running of $\alpha$. The "negative" $E$ axis is assigned to space-like momentum transfer. In the time-like region the resonances lead to pronounced variations of the effective charge.

uncertainty of $\delta \Delta \alpha\left(M_{Z}\right)=0.00036$ leads to an error $\delta \sin ^{2} \Theta_{\text {eff }}=0.00013$ in the prediction of $\sin ^{2} \Theta_{\text {eff }}$, and affects the Higgs mass bound, precision tests and new physics searches. Note that once $m_{H}$ will have been measured by the LHC $\sin ^{2} \Theta_{\text {eff }}$ will be an excellent monitor for new physics! This will be particularly important once the top mass $m_{t}$ will have been determined with higher accuracy.

One also should keep in mind that for calculations of perturbative QCD contributions precise QCD parameters $\alpha_{s}, m_{c}, m_{b}, m_{t}$ are mandatory.

\section{UPDATED EVALUATION OF $\alpha\left(M_{Z}\right)$}

Since my last major update in August 2006 a number of new results mainly from BaBar [4] were published. In fact a series of new channels have been measured in a range which covers the problematic region between 1.4 and $2.4 \mathrm{GeV}$. This means that we have almost completely new data for the exclusive measurements in this region. In contrast the inclusive measurements date back to the early 1980's. Important new cross-section measurements were also presented by KLOE at this meeting [5] (see also status reports from CMD-2/SND, BaBar, Belle, CLEO and BES at this meeting). The standard evaluation of the non-perturbative hadronic contributions in terms of measured cross-sections $\sigma\left(e^{+} e^{-} \rightarrow\right.$ hadrons) is based on the dispersion integral:

$\Delta \alpha_{\text {had }}^{(5)}(s)=-\frac{\alpha s}{3 \pi}\left(\oiint_{4 m_{\pi}^{2}}^{E_{\text {cut }}^{2}} d s^{\prime} \frac{R_{\gamma}\left(s^{\prime}\right)}{s^{\prime}\left(s^{\prime}-s\right)}\right)$,

where the $e^{+} e^{-}$-data are encoded in $R_{\gamma}(s) \equiv$ $\sigma^{(0)}\left(e^{+} e^{-} \rightarrow \gamma^{*} \rightarrow\right.$ hadrons $) / \frac{4 \pi \alpha^{2}}{3 s}$.

The evaluation of the integral at $M_{Z}=91.19$ $\mathrm{GeV}$ is performed by using $R(s)$ data up to $\sqrt{s}=$ $E_{\text {cut }}=5 \mathrm{GeV}$ and for the $\Upsilon$ resonances region between 9.6 and $13 \mathrm{GeV}$. Perturbative QCD is applied from 5.0 to $9.6 \mathrm{GeV}$ and for the high energy tail above $13 \mathrm{GeV}$. The result is

$$
\begin{aligned}
\Delta \alpha_{\text {hadrons }}^{(5)}\left(M_{Z}^{2}\right) & =0.027594 \pm 0.000219 \\
\alpha^{-1}\left(M_{Z}^{2}\right) & =128.946 \pm 0.030 .
\end{aligned}
$$

Note that BaBar exclusive radiative return measurements in this evaluation play an essential role up to $2 \mathrm{GeV}$ [lower end of BES inclusive measurement]. In the problematic region from 1.4 to $2 \mathrm{GeV}$ the exclusive measurements actually dominate in comparison to the much older inclusive measurements from Frascati [MEA, $\gamma \gamma 2$, M3N, $\mathrm{B} \overline{\mathrm{B}}]$. More detail are given in Table 1.

\section{TESTING NON-PERTURBATIVE HADRONIC EFFECTS VIA THE ADLER FUNCTION}

The non-perturbative Adler function related to the photon vacuum polarization can be calculated in terms of experimental $e^{+} e^{-}$annihilation data by the dispersion integral

$$
\begin{aligned}
D\left(Q^{2}\right)=Q^{2} & \left(\int_{4 m_{\pi}^{2}}^{E_{\text {cut }}^{2}} \frac{R^{\mathrm{data}}(s)}{\left(s+Q^{2}\right)^{2}} d s\right. \\
& \left.+\int_{E_{\text {cut }}^{2}}^{\infty} \frac{R^{\mathrm{pQCD}}(s)}{\left(s+Q^{2}\right)^{2}} d s\right) .
\end{aligned}
$$

Here $Q^{2}=-q^{2}$ is the squared Euclidean momentum transfer and $s$ the center of mass en- 
Table 1

Contributions and uncertainties for $\Delta \alpha_{\text {had }}^{(5)}\left(M_{Z}^{2}\right)^{\text {data }} \times 10^{4}$.

\begin{tabular}{ccrrrr}
\hline final state & range $(\mathrm{GeV})$ & \multicolumn{1}{c}{ result (stat) $($ syst $)[$ tot] } & \multicolumn{1}{c}{ rel } & \multicolumn{1}{c}{ abs } \\
\hline$\rho$ & $(0.28,0.81)$ & $25.95(0.09)(0.14)[0.17]$ & $0.6 \%$ & $0.6 \%$ \\
$\omega$ & $(0.42,0.81)$ & $2.91(0.03)(0.08)[0.09]$ & $3.0 \%$ & $0.2 \%$ \\
$\phi$ & $(1.00,1.04)$ & $4.42(0.06)(0.10)[0.12]$ & $2.7 \%$ & $0.3 \%$ \\
$J / \psi$ & & $11.14(0.53)(0.58)[0.79]$ & $7.1 \%$ & $12.9 \%$ \\
$\Upsilon$ & & $1.18(0.05)(0.06)[0.08]$ & $6.9 \%$ & $0.1 \%$ \\
had & $(0.81,1.40)$ & $13.21(0.04)(0.35)[0.35]$ & $2.7 \%$ & $2.6 \%$ \\
had & $(1.40,2.00)$ & $11.34(0.07)(1.26)[1.26]$ & $11.2 \%$ & $33.3 \%$ \\
had & $(2.00,3.10)$ & $15.73(0.11)(0.87)[0.88]$ & $5.6 \%$ & $16.2 \%$ \\
had & $(3.10,3.60)$ & $5.26(0.11)(0.10)[0.15]$ & $2.8 \%$ & $0.5 \%$ \\
had & $(3.60,9.46)$ & $50.58(0.11)(0.24)[0.26]$ & $0.5 \%$ & $1.5 \%$ \\
had & $(9.46,13.00)$ & $18.52(0.25)(1.21)[1.23]$ & $6.7 \%$ & $31.8 \%$ \\
pQCD & $(13.0, \infty)$ & $115.71(0.00)(0.06)[0.06]$ & $0.0 \%$ & $0.1 \%$ \\
\hline data & $(0.28,13.00)$ & $160.23(0.63)(2.10)[2.19]$ & $1.4 \%$ & $0.0 \%$ \\
total & & $275.94(0.63)(2.10)[2.19]$ & $0.8 \%$ & $100.0 \%$ \\
\hline
\end{tabular}

ergy squared for hadron production in $e^{+} e^{-}-$ annihilation. Formally the Adler function is defined as the derivative of the shift in the fine structure constant

$$
\frac{D\left(Q^{2}\right)}{Q^{2}}=\left(12 \pi^{2}\right) \frac{d \Pi_{\gamma}^{\prime}\left(q^{2}\right)}{d q^{2}}=-\frac{3 \pi}{\alpha} \frac{d}{d q^{2}} \Delta \alpha_{\mathrm{had}}\left(q^{2}\right)
$$

evaluated in the Euclidean at $Q^{2}=-q^{2} . \Pi_{\gamma}^{\prime}\left(q^{2}\right)$ is the photon vacuum polarization amplitude defined by

$$
\begin{aligned}
\Pi_{\mu \nu}^{\gamma}(q) & =i \int d^{4} x e^{i q x}<0\left|T J_{\mu}^{\gamma}(x) J_{\nu}^{\gamma}(0)\right| 0> \\
& =-\left(q^{2} g_{\mu \nu}-q_{\mu} q_{\nu}\right) \Pi_{\gamma}^{\prime}\left(q^{2}\right) .
\end{aligned}
$$

The perturbative result is given in [7]. Crucial for this prediction are known full massive QCD results [10-12]. Note that the main $Q^{2}$ dependence of $D\left(Q^{2}\right)$ is due to the quark masses $m_{c}$ and $m_{b}$. Without mass effects, up to small effects from the running of $\alpha_{s}, D\left(Q^{2}\right)=3 \sum_{f} Q_{f}^{2}\left(1+O\left(\alpha_{s}\right)\right)$ is a constant depending on the number of active flavors. We also include the 4-loop [13,14] and 5-loop [15] contributions in the high energy limit (massless approximation)

$$
D\left(Q^{2}\right) \simeq 3 \sum_{f} Q_{f}^{2}\left(1+a+d_{2} a^{2}+d_{3} a^{3}+d_{4} a^{4}\right)
$$

with $a=\alpha_{s}\left(Q^{2}\right) / \pi, d_{2}=1.9857-0.1153 n_{f}$, $d_{3}=18.2428-4.2159 n_{f}+0.0862 n_{f}^{2}-$ $1.2395\left(\sum Q_{f}\right)^{2} /\left(3 \sum Q_{f}^{2}\right)$ and $d_{4}=-0.010 n_{f}^{3}+$ $1.88 n_{f}^{2}-34.4 n_{f}+135.8$. The corresponding formula for $R(s)$ only differs at the $4-$ loop and $5-$ loop level due to the effect from the analytic continuation from the Euclidean to the Minkowski region which yields $r_{3}^{R}=d_{3}-\pi^{2} \beta_{0}^{2} \frac{d_{1}}{3}$ with $\beta_{0}=\left(11-2 / 3 n_{f}\right) / 4, d_{1}=1$ and $r_{4}^{R}=d_{4}-$ $\pi^{2} \beta_{0}^{2}\left(d_{2}+\frac{5 \beta_{1}}{6 \beta_{0}} d_{1}\right)$ with $\beta_{1}=\left(102-38 / 3 n_{f}\right) / 16$. Numerically the 4 -loop term proportional to $d_{3}$ amounts to $-0.0036 \%$ at $100 \mathrm{GeV}$ and increases to about $0.32 \%$ at $2.5 \mathrm{GeV}$. The higher order massless results only improve the perturbative high energy tail (see Fig. 2). Towards low $Q^{2}$ we also approach the Landau pole of $\alpha_{s}\left(Q^{2}\right)$, present typically in $\overline{\mathrm{MS}}$ type schemes, and pQCD ceases to "converge".

\section{5. $\Delta \alpha^{\text {had }}$ VIA THE ADLER FUNCTION}

Figure 2 provides convincing evidence that pQCD works well to predict $D\left(Q^{2}\right)$ down to $Q \sim M_{0}=2.5 \mathrm{GeV}$. This may be used to calculate

$$
\Delta \alpha_{\text {had }}\left(-Q^{2}\right) \sim \frac{\alpha}{3 \pi} \int d Q^{\prime 2} \frac{D\left(Q^{\prime 2}\right)}{Q^{\prime 2}}
$$

and we may write

$$
\Delta \alpha_{\text {had }}^{(5)}\left(M_{Z}^{2}\right)=\Delta \alpha_{\text {had }}^{(5)}\left(-M_{0}^{2}\right)^{\text {data }}
$$




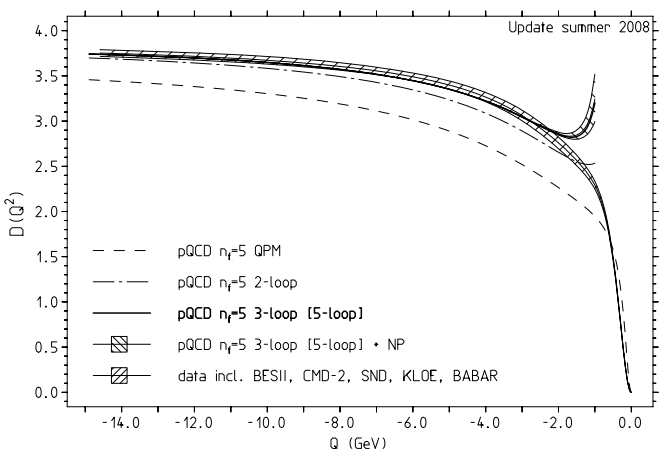

Figure 2. The "experimental" non-perturbative Adler-function versus theory (pQCD + NP). The error includes statistical + systematic here (in contrast to most $R$-plots showing statistical errors only!). "[5-loop]" indicates that 4- and 5loop contribution in the massless limit are taken into account. For more details see Ref. [7].

$$
\begin{aligned}
& +\left[\Delta \alpha_{\text {had }}^{(5)}\left(-M_{Z}^{2}\right)-\Delta \alpha_{\text {had }}^{(5)}\left(-M_{0}^{2}\right)\right]^{\mathrm{pQCD}} \\
& +\left[\Delta \alpha_{\text {had }}^{(5)}\left(M_{Z}^{2}\right)-\Delta \alpha_{\text {had }}^{(5)}\left(-M_{Z}^{2}\right)\right]^{\mathrm{pQCD}}
\end{aligned}
$$

and obtain, for $M_{0}=2.5 \mathrm{GeV}$

$$
\begin{aligned}
\Delta \alpha_{\text {had }}^{(5)}\left(-M_{0}^{2}\right)^{\text {data }} & =0.007354 \pm 0.000107 \\
\Delta \alpha_{\text {had }}^{(5)}\left(-M_{Z}^{2}\right) & =0.027477 \pm 0.000149 \\
\Delta \alpha_{\text {had }}^{(5)}\left(M_{Z}^{2}\right) & =0.027515 \pm 0.000149
\end{aligned}
$$

where a tiny shift of +0.000008 results from the 5-loop contribution. An error \pm 0.000103 added in quadrature comes form the perturbative part. For the perturbative calculation of $\left[\Delta \alpha_{\text {had }}^{(5)}\left(-M_{Z}^{2}\right)-\Delta \alpha_{\text {had }}^{(5)}\left(-M_{0}^{2}\right)\right]^{\mathrm{pQCD}}$ we use the QCD parameters: $\alpha_{s}\left(M_{Z}\right)=0.1189(20)$,

$$
m_{c}\left(m_{c}\right)=1.286(13)\left[M_{c}=1.666(17)\right] \mathrm{GeV},
$$$$
m_{b}\left(m_{c}\right)=4.164(25)\left[M_{b}=4.800(29)\right] \mathrm{GeV} \text {, }
$$

based on a complete 3-loop massive QCD analysis [16,17] (see contributions by Kühn and Sturm). Note that due to a dramatic improvement in the determination of the quark masses $m_{c}$ and $m_{b}$, for the first time the pQCD error included in (8) is smaller than the one from the data which also has been improved substantially. A very important long term project here is the

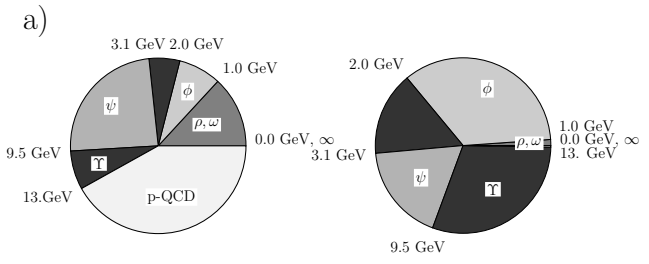

b)
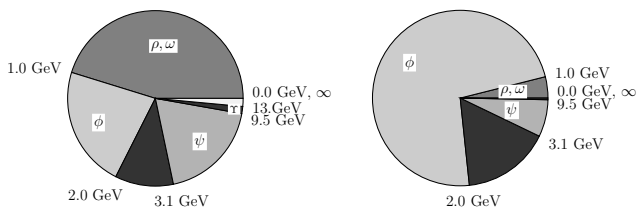

Figure 3. Present distribution of contributions [left] and errors ${ }^{2}$ [right] for a) $\Delta \alpha_{\text {hadrons }}^{(5)}\left(M_{Z}^{2}\right)$; b) $\Delta \alpha_{\text {had }}^{(5)}\left(-M_{0}^{2}\right)^{\text {data }}\left(M_{0}=2.5 \mathrm{GeV}\right)$; both obtained by direct integration of (2).

lattice determinations of the basic QCD parameters [18].

Mandatory pQCD improvements required are: - 4-loop massive pQCD calculation of Adler function; required are a number of terms in the low and high momentum series expansions which allow for the appropriate Padé improvements [essentially equivalent to a massive 4-loop calculation of $R(s)$ ];

- $m_{c}$ improvement by sum rule and/or lattice QCD evaluations;

- improved $\alpha_{s}$ in low $Q^{2}$ region.

Renormalization schemes which exhibit a Landau pole, like the $\overline{\mathrm{MS}}$ scheme, evidently fail in parametrizing the low energy tail of the Adler function. Therefore modeling the Adler function at low $Q^{2}$ by testable models may be useful, such as "analytized" $\alpha_{s}$ [19] and the instanton liquid model [20] or - others.

The contribution and error profiles of $\Delta \alpha_{\text {had }}^{(5)}\left(M_{Z}^{2}\right)$ and $\Delta \alpha_{\text {had }}^{(5)}\left(-M_{0}^{2}\right)$, are shown in Fig. 3. Fig. 4 illustrates where more precise measurements are particularly important. For the Adler function approach in particular low energy machines below $2.5 \mathrm{GeV}$ most successfully can contribute to improve the precise determination of $\alpha(E)$. At the same time machines in this regime substantially contribute to further reduce the error of the leading hadronic contribution to the muon $g-2$. 
Table 2

Contributions and uncertainties for $\Delta \alpha_{\text {had }}^{(5)}\left(-M_{0}^{2}\right)^{\text {data }} \times 10^{4}\left(M_{0}=2.5 \mathrm{GeV}\right)$.

\begin{tabular}{ccrrrr}
\hline final state & range $(\mathrm{GeV})$ & \multicolumn{1}{c}{ result (stat) (syst) [tot] } & rel & abs \\
\hline$\rho$ & $(0.28,0.81)$ & $24.06(0.09)(0.13)[0.16]$ & $0.6 \%$ & $2.1 \%$ \\
$\omega$ & $(0.42,0.81)$ & $2.65(0.03)(0.07)[0.08]$ & $3.0 \%$ & $0.5 \%$ \\
$\phi$ & $(1.00,1.04)$ & $3.79(0.05)(0.09)[0.10]$ & $2.7 \%$ & $0.9 \%$ \\
$J / \psi$ & & $3.95(0.19)(0.18)[0.26]$ & $6.6 \%$ & $5.9 \%$ \\
$\Upsilon$ & & $0.07(0.00)(0.00)[0.00]$ & $6.7 \%$ & $0.0 \%$ \\
had & $(0.81,1.40)$ & $11.33(0.03)(0.29)[0.29]$ & $2.6 \%$ & $7.3 \%$ \\
had & $(1.40,2.00)$ & $7.81(0.05)(0.87)[0.87]$ & $11.2 \%$ & $65.8 \%$ \\
had & $(2.00,3.10)$ & $7.91(0.05)(0.44)[0.44]$ & $5.6 \%$ & $16.7 \%$ \\
had & $(3.10,3.60)$ & $1.88(0.04)(0.04)[0.05]$ & $2.8 \%$ & $0.2 \%$ \\
had & $(3.60,9.46)$ & $8.11(0.02)(0.05)[0.05]$ & $0.6 \%$ & $0.2 \%$ \\
had & $(9.46,13.00)$ & $0.89(0.01)(0.06)[0.06]$ & $6.6 \%$ & $0.3 \%$ \\
pQCD & $(13.0, \infty)$ & $1.09(0.00)(0.00)[0.00]$ & $0.1 \%$ & $0.0 \%$ \\
\hline data & $(0.28,13.00)$ & $72.45(0.23)(1.05)[1.08]$ & $1.5 \%$ & $0.0 \%$ \\
total & & $73.54(0.23)(1.05)[1.08]$ & $1.5 \%$ & $100.0 \%$ \\
\hline
\end{tabular}
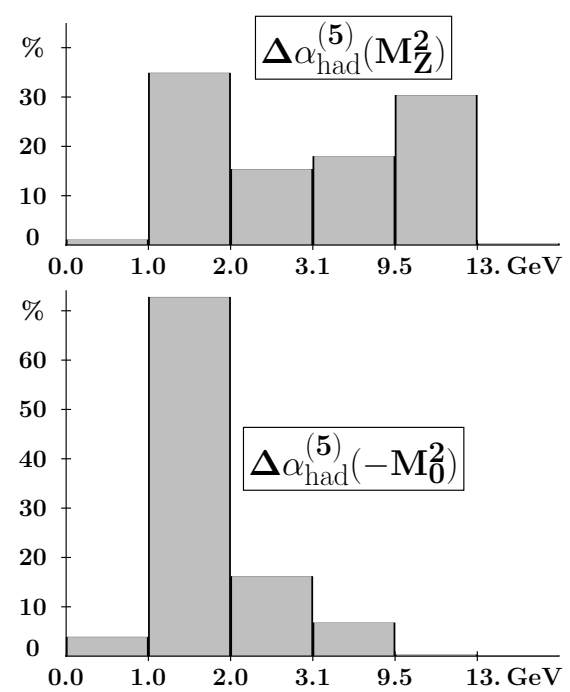

Figure 4. Present error profiles for $\Delta \alpha_{\text {had }}^{(5)}\left(M_{Z}^{2}\right)$ and $\Delta \alpha_{\text {had }}^{(5)}\left(-M_{0}^{2}\right)$.

\section{POSSIBLE IMPROVEMENT BY VEPP-2000 and DAFNE-2}

Next generation precision physics experiments, not only the ones possible at an ILC, in many cases require a more precise determination of $\alpha(E)$. A reasonable goal could be an improve- ment by about a factor 10 in accuracy which would match the precision of the $Z$ mass. The options are

- the standard approach by direct integration of the $e^{+} e^{-}$-data: in this case $58 \%$ of the contribution is obtained from the data and $42 \%$ from pQCD. My analysis yields $\Delta \alpha_{\text {had }}^{(5) \text { data }} \times 10^{4}=$ $160.12 \pm 2.24(1.4 \%)$ and thus increasing the overall accuracy to $1 \%$ would yield an uncertainty \pm 1.63 . However, for independent measurements in ranges as used in the Tab. 1 a $1 \%$ accuracy for each region and errors including systematic ones added in quadrature would yield \pm 0.85 . The improvement on the data $([2.24]$ vs. [0.85]) thus would yield an improvement factor of 2.6. The pQCD part in this case is $\Delta \alpha_{\text {had }}^{(5) \mathrm{pQCD}} \times 10^{4}=$ $115.71 \pm 0.06(0.05 \%)$ and for the theory part this means that no improvement would be needed.

- With the "Adler function approach" we get $26 \%$ of the contribution from data and $74 \%$ from pQCD. Here $\Delta \alpha_{\text {had }}^{(5) \text { data }} \times 10^{4}=72.35 \pm 1.10$ $(1.5 \%)$ and a $1 \%$ overall accuracy would mean an uncertainty \pm 0.74 . Again, a subdivision of ranges as used in Tab. 2 and assuming that a $1 \%$ accuracy can be reached for each region and adding up errors in quadrature in this case would lead to a precision of \pm 0.40 . The improvement from the data ([1.10] vs. [0.40]) again yields a 
similar improvement factor of 2.7 . If we compare the standard approach of direct integration with the Adler function controlled approach ([2.24] vs. [0.40]) we have an improvement factor 5.6. However, now a much larger fraction $\Delta \alpha_{\text {had }}^{(5) \mathrm{pQCD}} \times 10^{4}=201.15 \pm 1.03$ is coming from pQCD and an improvement of the QCD prediction is mandatory in order to profit in an optimal way from the improvement on the data. A factor 3 to 5 at least should be possible in a long term effort on higher order effects and more importantly on QCD parameters. An accuracy of about \pm 0.20 would be a high goal.

Our study shows that the requirement Eq. (1) could be achieved by

- pinning down experimental errors to the $1 \%$ level in all non-perturbative regions up to $10 \mathrm{GeV}$

- safely use pQCD in the Euclidean region monitored by the Adler function

- improve on pQCD and QCD parameters.

In any case as we see from Fig. 4 by far the largest improvement factor will come from precise crosssection measurements in the region from 1.4 to 2.4 $\mathrm{GeV}$. A unique challenge and chance for VEPP2000 and DAFNE-2.

Acknowledgments: I am very grateful to the organizers of the Frascati Int. Workshop " $e^{+} e^{-}$ Collisions from $\Phi$ to $\Psi$ " for the kind invitation and for support. It was a very interesting and inspiring event. I also thank S. Jadach and his team from the Niewodniczanski Nuclear Physics Institute at Krakow for the kind hospitality extended to me.

\section{REFERENCES}

1. B. Khazin, these proceedings; S. Eidelman, Nucl. Phys. Proc. Suppl. 162 (2006) 323.

2. P. Raimondi, these proceedings; G. Venanzoni, Acta Phys. Polon. B 38 (2007) 3421; F. Ambrosino et al., Eur. Phys. J. C 50 (2007) 729.

3. F. Jegerlehner, The effective fine structure constant at TESLA energies, hepph/0105283.

4. B. Aubert et al. [BABAR Collab.], Phys. Rev. D 76 (2007) 012008; Phys. Rev. D 76 (2007) 092005; Phys. Rev. D 76 (2007) 092006;
arXiv:0710.4451 [hep-ex].

5. F. Nguyen [for the KLOE Collaboration], these proceedings; arXiv:0807.1612 [hep-ex].

6. F. Jegerlehner, Nucl. Phys. Proc. Suppl. 162 (2006) 22.

7. S. Eidelman, F. Jegerlehner, A. L. Kataev, O. Veretin, Phys. Lett. B 454 (1999) 369.

8. F. Jegerlehner, In: Radiative Corrections, ed by J. Solà (World Scientific, Singapore 1999) pp 75-89.

9. S. Eidelman, F. Jegerlehner, Z. Phys. C 67 (1995) 585; F. Jegerlehner, Nucl. Phys. (Proc. Suppl.) C 51 (1996) 131; J. Phys. G 29 (2003) 101; Nucl. Phys. Proc. Suppl. 126 (2004) 325.

10. K. G. Chetyrkin, J. H. Kühn, M. Steinhauser, Nucl. Phys. B 482 (1996) 213; Nucl. Phys. B 505 (1997) 40.

11. K. G. Chetyrkin, R. Harlander, J. H. Kühn, M. Steinhauser, Nucl. Phys. B 503 (1997) 339.

12. F. Jegerlehner, O. V. Tarasov, Nucl. Phys. B 549 (1999) 481.

13. S. G. Gorishnii, A. L. Kataev, S. A. Larin, Phys. Lett. B 259 (1991) 144.

14. L. R. Surguladze, M. A. Samuel, Phys. Rev. Lett. 66 (1991) 560 [Erratum-ibid. 66 (1991) 2416].

15. J. H. Kühn, these proceedings; P. A. Baikov, K. G. Chetyrkin, J. H. Kühn, Phys. Rev. Lett. 88 (2002) 012001; Phys. Rev. D 67 (2003) 074026; Phys. Lett. B 559 (2003) 245; arXiv:0801.1821 [hep-ph].

16. C. Sturm, these preceedings; J. H. Kühn, M. Steinhauser, C. Sturm, Nucl. Phys. B 778 (2007) 192.

17. R. Boughezal, M. Czakon, T. Schutzmeier, Phys. Rev. D 74 (2006) 074006.

18. J. Heitger, these proceedings; M. Della Morte, N. Garron, M. Papinutto, R. Sommer, JHEP 0701 (2007) 007; G. M. de Divitiis, M. Guagnelli, R. Petronzio, N. Tantalo, F. Palombi, Nucl. Phys. B 675 (2003) 309; J. Rolf, S. Sint [ALPHA Collab.], JHEP 0212 (2002) 007.

19. D. V. Shirkov, I. L. Solovtsov, Theor. Math. Phys. 150 (2007) 132.

20. A. E. Dorokhov, Phys. Rev. D 70 (2004) 094011. 